\title{
Preface to Special Issue on Environmental and Energy Catalysis
}

Environmental pollution and energy storage are two major challenges faced by human beings. The magnitude of them is ever-increasing due to rapid pace of urbanization and industrialization. In view of this, to achieve green environment and provide clean energy for human beings are pivotal for sustainability. The catalysis technology plays dominant role in addressing these issues. The nano/microstructured catalyst with intriguing physical and chemical properties could offer numerous opportunities to realize environmental sustainability and clean energy production. In the past two decades, great advances have been made on the design, synthesis and mechanistic understanding of typical catalysts for environmental and energetic applications. These new catalysts in various fashions can be classified into three main types, thermal catalysis, photocatalysis and electro catalysis. In some cases, two types can be combined together, such as photoelectrocatalysis and photothermal catalysis, to achieve higher catalysis efficiency. The features of catalysts can be further tailored to allow for enhanced catalytic performance in pollutant degradation and energy conversion. Advanced in situ techniques have been applied to explore and reveal the catalytic mechanisms.

The recent rapid development in the area of catalysis for sustainability and energy inspired us to edit this special issue of Chinese Journal of Catalysis. We have invited more than thirty young scientists to contribute their research/review articles that have improved our understanding of the key scientific and technological problems in environmental and energy catalysis. Their contributions represent excellent examples of future development trend in this important field. Among them, three papers are review papers and others are research articles. All the published papers have been reviewed according to the normal review practices of Chinese Journal of Catalysis. These advances on catalysis could provide new insights into our understanding on the controlled synthesis, catalytic mechanism and structure-activity relationship of various catalysts for green environment and clean energy.
At last, as the Guest Editors of this special issue, we would like to express my sincere thanks to all the authors who contributed to this special issue and all the reviewers for their valuable comments on the submitted manuscripts. Especially, we appreciate the Editorial Office Staff of Chinese Journal of Catalysis for their hard work in editing and publishing this special issue.

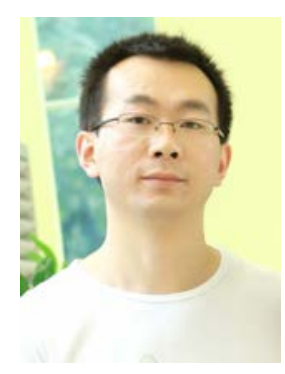

Prof. Fan Dong

Chongqing Key Laboratory of Catalysis and New Environmental Materials;

College of Environment and Resources, Chongqing Technology and Business University E-mail address: dfctbu@126.com

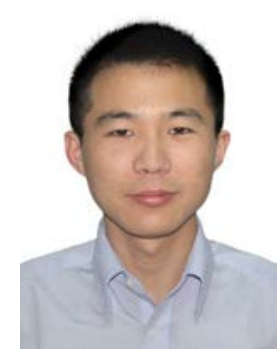

Prof. Jiguang Deng

Beijing Key Laboratory for Green Catalysis and Separation, College of Environmental and Energy Engineering, Beijing University of Technology E-mail address: jgdeng@bjut.edu.cn 